\title{
Praktiken des Sprechens und Interagierens im Gespräch aus der Sicht von Konversationsanalyse und Interaktionaler Linguistik ${ }^{1}$
}

\begin{abstract}
Der Beitrag erläutert zunächst die Verwendungsweise des Begriffs der ,Praktik(en)' im Rahmen der Konversationsanalyse und Interaktionalen Linguistik. Daran anschließend werden Beispiele der Herstellung und Kontextualisierung von emotional beteiligten Höhepunkten konversationeller Erzählungen untersucht, in denen die Erzähler/innen und Zuhörer/innen bestimmte Affekte erkennbar machen: Freude oder Belustigung in Belustigungserzählungen und Ärger oder Entrüstung in Beschwerdeerzählungen. Es wird gezeigt, dass die gleichen Praktiken verwendet werden, um die Interpretation dieser Affekte im sequenziellen Kontext der Erzählungen nahezulegen, dass sich aber die eingesetzten Ressourcen dabei je nach Affekt zumindest teilweise unterscheiden.
\end{abstract}

\section{Einleitung}

,Praktiken' werden auch in der Konversationsanalyse (CA) und Interaktionalen Linguistik (IL) untersucht. Generelles Ziel der Konversationsanalyse ist die Beschreibung der Methoden der Erzeugung von Ordnung und Sinn in der sozialen Interaktion, vor allem im Bereich der sequenziellen Organisation sprachlicher Interaktion (Sacks/Schegloff/Jefferson 1974; Schegloff 2007; vgl. auch Sidnell 2010). Aufbauend auf den Prämissen und Methoden der Konversationsanalyse fokussiert die Interaktionale Linguistik in zwei möglichen Herangehensweisen die beiden Fragen, wie einerseits soziale Interaktion mit sprachlichen (und anderen) Ressourcen hergestellt wird, und wie andererseits linguistische (und andere) Ressourcen in der sozialen Interaktion verwendet werden (Selting/Couper-Kuhlen 2001; Couper-Kuh-

1 Für ihre Kommentare zu einer früheren Version dieses Papiers danke ich den Teilnehmerinnen und Teilnehmern des ,Kolloquiums zur linguistischen Kommunikationsforschung an der Universität Potsdam, vor allem Dagmar Barth-Weingarten, Bernhard Bielick, Uwe Küttner, Maxi Kupetz, Jana Scheerer, Yuko Sugita und Fabienne Tissot. Außerdem danke ich Yuko Sugita für Hilfe bei den Praat-Analysen, Maxi Kupetz für Hilfe bei der Herstellung der Standbilder. 
len/Selting 2001, i.Vorb.). Dabei wird das Zusammenspiel verbaler, vokaler und, soweit relevant, visueller Ressourcen in den Blick genommen (vgl. Selting 2013).

In der Denkweise der CA und IL steht der Begriff der ,Praktiken“ in einem engen Zusammenhang mit anderen Begriffen, die für die Analyse des Handelns in der sozialen Interaktion gebraucht werden. Wir arbeiten mit folgenden Annahmen und Begriffen:

- Interaktionspartner interagieren miteinander, um soziale Handlungen und Aktivitäten zu vollziehen. Eine ,Handlung، (action) ist dabei nach Levinson (2013, S. 107) das, was der ,main job' eines Turns ist, womit die Reaktion sich befassen muss, um als adäquater nächster Turn zu gelten. ,Aktivitäten‘ (activities) sind demgegenüber größere und komplexere Handlungen, ,big packages‘, wie z.B. Erzählen, Argumentieren, Beschreiben; das, was im deutschen Forschungskontext auch als ,kommunikative Gattungen' beschrieben wird (vgl. Günthner/Knoblauch 1994; Günthner 2000). ${ }^{2}$

Alle Details des Sprechens und sonstigen Miteinander-Umgehens der Interaktionspartner sind darauf angelegt, Turns (Gesprächsbeiträge) und Turnkonstruktionseinheiten (beitragsbildende Einheiten), zugeschnitten auf den/die Rezipienten, die sequenzielle Position und den Situationskontext, so zu produzieren, dass die damit ausgeführte Handlung erkennbar wird und der Rezipient entsprechend reagieren kann. Hierfür sind ,Praktiken' und ,Ressourcen' relevant.

Die Unterscheidung zwischen ,Handlungen' (actions) und ,Praktiken' (practices) wurde v.a. von Schegloff (1997) fokussiert und explizit gemacht. Er untersucht die Verbindungen zwischen ,practices of talk-in-interaction and the actions which they accomplish“ (ebd., S. 499; vgl. auch ebd., S. 539). Das heißt, Praktiken werden verwendet, um damit Handlungen auszuführen. ${ }^{3}$

Als Beispiel verweist Schegloff auf Praktiken des Sprechens, die üblicherweise verwendet werden, um Reparaturen zu initiieren. Im Englischen wären das z.B. das Signal huh?, Fragewörter wie who? usw., und bestimmte Arten von Wiederholungen (repeats). Wenn solche Formen oder Formate zur Initiierung von Reparaturen verwendet werden, dann wird das als Praktik beschrieben.

2 Die Unterscheidung zwischen Handlungen und Aktivitäten bzw. kommunikativen Gattungen differenziert im Bereich dessen, was in älteren Arbeiten noch undifferenziert je nach Forscher/ Forscherin entweder als sprachliche Handlungen oder als Aktivitäten bezeichnet wurde. Gegenüber dem Begriff der Aktivitäten hebt der Begriff der kommunikativen Gattungen stärker die Vorgeformtheit und Verfestigung von komplexeren Handlungsabläufen hervor (Günthner/Knoblauch 1994).

3 Allerdings wird der Begriff Praktik (practices) in der CA nicht ganz einheitlich verwendet. Siehe dazu Levinson (2013, S. 129, Fußnote 9). 
Jedoch können Praktiken, die mit der routinemäßigen Initiierung von Reparaturen assoziiert sind, auch für andere Zwecke benutzt werden. Schegloff gibt folgende Beispiele: huh? oder hm? können auch eingesetzt werden, um nach einer Frage eine zunächst ausbleibende Antwort noch einmal relevant zu machen, oder um eine Erzählung hervorzulocken oder voranzutreiben (vgl. 1997, S. 508 ff.). Diese Beobachtung motiviert die weitere Unterscheidung zwischen der ,Praktik als routinemäßiger Verwendung der sprachlichen Einheiten, und den sprachlichen Einheiten selbst: den ,Ressourcen', d.h. ,Formen' oder ,Formate', die im Beispielfall der Reparaturinitiierung aus den Partikeln huh?, hm? usw. bestehen, die aber auch für andere Aufgaben verwendet werden können. Der Begriff Praktik soll betonen, dass Sprecher/innen und Handelnde mit der Verwendung von sprachlichen Einheiten und Signalen etwas tun, und dass Rezipient/inn/en sie verstehen im Hinblick auf das, was sie tun (vgl. ebd., S. 539; siehe auch Heritage 2010).

Wenn wir in der IL diese Unterscheidungen übernehmen, dann fassen wir Praktiken als Verbindung auf zwischen, auf der einen Seite, Ressourcen, d.h. Formen und Formaten, und, auf der anderen Seite, Handlungen: Durch die Verwendung einer für eine bestimmte Handlung designierten Praktik wird diese bestimmte Handlung ausgeführt, deren Erkennen ermöglicht oder deren Interpretation nahegelegt. Die Praktik selbst wird hergestellt durch die rekurrente Verwendung und Kombination bestimmter Ressourcen oder Ressourcenbündel, d.h. sprachliche und nicht-sprachliche Formen und Formate, für die Herstellung und Kontextualisierung einer bestimmten Handlung in einem bestimmten sequenziellen Kontext.

Diese Ausführungen haben deutlich gemacht, dass Praktiken bestimmte generelle Eigenschaften haben: Sie sind kontextsensitiv, also routinisierte Verwendungsweisen von Ressourcen für situierte Handlungen, die flexibel an die je spezifischen Gegebenheiten angepasst werden. Sie sind immer eingebettet in soziale Handlungszusammenhänge, in deren Kontext sie ihre Funktion, ihren Sinn und ihre Interpretation für die Interaktionspartner gewinnen. Sie werden von Interaktionspartnern in ihren spezifischen Beteiligungsrollen verwendet bzw. verstanden, sind also an eine bestimmte Beteiligungsstruktur gebunden (participant framework). Das werden auch die im Folgenden dargestellten Beispielanalysen zeigen (vgl. dazu auch Deppermann/Feilke/Linke in diesem Band).

Im Folgenden werde ich zwei Beispiele untersuchen, in denen Praktiken der Nahelegung (der Interpretation) emotionaler Beteiligung verwendet werden zur Kontextualisierung von Höhepunkten konversationeller Erzählungen. Ich werde zeigen, dass innerhalb der Aktivität konversationelles Erzählen für die Handlung der Kontextualisierung von Höhepunkten freudiger oder belustigender und ärgerlicher oder Beschwerdeerzählungen die gleichen Praktiken verwendet, für die Darstellung der jeweiligen Affekte aber z.T. andere Ressourcen eingesetzt werden. 


\section{Praktiken der Kontextualisierung von Höhepunkten von Belustigungs- und Beschwerdeerzählungen}

Erzählungen sind komplexe Aktivitäten, bei denen Erzähler eine Ereigniskette zugeschnitten auf ihre Rezipienten und den Kontext des Erzählens darbieten und dabei verschiedene Teile ihrer Erzählung erkennbar machen, damit die Rezipienten die Erzählung verstehen und adäquat reagieren können. Dazu gehört, dass der/die Erzähler/in sich in einer Präsequenz aus Ankündigung und Ratifizierung die Zuhörer als Mit-Produzenten des Erzählens sichert, eine Ereigniskette darstellt, in der die angekündigten Ereignisse elaboriert und/oder dramatisiert werden, und schließlich die Erzählung abschließt und ins übergeordnete Gespräch zurückleitet (vgl. auch Sacks 1971, 1986; Jefferson 1978; Goodwin 1984, 1997; Selting 1995b; Quasthoff 2001).

In vielen Fällen kulminiert die Elaboration der Ereigniskette in einem Höhepunkt der Erzählung, an dem zugleich auch erhöhte emotionale Beteiligung gezeigt wird. Das Kontextualisieren des Höhepunktes einer Erzählung macht eine Reaktion des Rezipienten relevant, je nach gezeigter Emotion muss diese Reaktion angepasst sein. Als traurig angekündigte Erzählungen verlangen nach mitfühlenden Reaktionen, als freudig oder belustigend angekündigte Erzählungen verlangen Lachen und Mit-Freude usw. Begrifflich werde ich allgemein von ,emotionaler Beteiligung' sowie spezifischer vom Nahelegen der Interpretation der Affekte ,Freude‘, ,Belustigung oder ,Ärger', ,Entrüstung' sprechen. ${ }^{4}$

Oft ist es aber gar nicht der Inhalt, der ein Ereignis als einen Höhepunkt des Erzählens ausmacht, sondern vielmehr dessen Präsentation mithilfe von Praktiken, in denen verbale, vokale und visuelle Ressourcen kombiniert werden, um es als Höhepunkt erkennbar zu machen. Nach einer entsprechenden Ankündigung sind so kontextualisierte Höhepunkte dann konstitutive Teile der Erzählung, die die Zuhörer erwarten, um ihre Reaktionen einzubringen und damit die Erzählung

\footnotetext{
4 Ich lehne mich an die Definition von Ochs/Schieffelin (1989, S. 7) an, die den Begriff ,Affekt als weiteren, übergeordneten festlegen, der emotionale Beteiligung im weiteren Sinne umfasst, d.h. ,emotions' (,Emotionen') - einschließlich ,basic emotions', ,feelings' (,Gefühle'), ,moods (,Stimmungen', ,Launen'), ,dispositions` (,Veranlagungen', ,Dispositionen') und ,attitudes` (,Einstellungen') (vgl. ebd.). Vieles davon ist in jüngster Zeit auch unter den Begriff, stance (,Haltung') subsumiert worden (vgl. Stivers 2008; Goodwin/Goodwin 2000). Manche Autoren verwenden die Begriffe ,stance ${ }^{*}$ und ,affect auch mehr oder weniger synonym oder als kombiniertes Begriffspaar. Ich stimme mit Local/Walker (2008) überein, den Begriff ,stance‘ als den generelleren Begriff zu verwenden (vgl. ebd., S. 745), der ,affect' einschließt.
} 
mitzukonstruieren. Die Verwendung geeigneter Praktiken durch den/die Erzähler/in, um diese Höhepunkte erkennbar zu machen, ist also eine Voraussetzung dafür, dass die Rezipienten richtig reagieren und damit die Erzählung mit-produzieren und zu einem unproblematischen Abschluss bringen können.

Emotionale Beteiligung wird bei der Kontextualisierung von Höhepunkten konversationeller Erzählungen mit unterschiedlichen Bezügen erkennbar gemacht: mit Bezug auf rekonstruierte Affekte in der Erzählwelt ebenso wie mit Bezug auf in-situ Affekte im Hier und Jetzt der Erzählsituation (vgl. Günthner 2000). Die beschriebenen Affekte werden immer als ,Darstellungen', als display, aufgefasst; sie müssen nicht unbedingt mit real gefühlten Emotionen übereinstimmen. Ich werde auch von ,Praktiken des Affektdisplay' reden.

Das Management von Höhepunkten konversationeller Erzählungen unterscheidet sich danach, ob die Rezipienten mit Affiliation reagieren oder nicht. Nach Stivers (2008, S. 35) meint affiliation, ,that the hearer displays support of and endorses the teller's conveyed stance", d.h. dass der Zuhörer Bestätigung, Befürwortung und Unterstützung der vom Erzähler vermittelten Haltung signalisiert. Wenn Rezipienten affiliative Reaktionen zeigen, stellen sie damit unproblematische Kooperativität her. Nicht-affiliative Reaktionen der Rezipienten führen dagegen zu weiteren Handlungen der Erzählerin mit verstärkten Affektausdrücken, mit denen sie die Affiliation des Zuhörers und damit unproblematische Kooperativität doch noch zu erreichen sucht. Ich werde zeigen, (1) mit welchen Praktiken im sequenziellen Kontext der Aktivität Erzählen die Erzähler/innen ihre Höhepunkte und ihre affektive Haltung erkennbar machen und damit passende affiliative Reaktionen der Rezipient/inn/en relevant machen, und (2) wie die Rezipienten auf diese Höhepunkte reagieren und damit den weiteren Verlauf der Aktivität Erzählen mitgestalten.

\subsection{Ziele und Methodologie der Beispielanalysen}

Meine Analyse basiert auf einem Korpus von Videoaufnahmen natürlicher Gespräche, das vom Projekt „Emotive involvement in conversational storytelling“ im Rahmen des Exzellenzclusters „Languages of Emotion“ an der FU Berlin erhoben wurde (Leitung: Elizabeth Couper-Kuhlen und Margret Selting). Die Daten wurden nach GAT2 (vgl. Selting et al. 2009) transkribiert; eine Zusammenstellung der wichtigsten Notationskonventionen findet sich im Anhang.

Meine Methodologie kombiniert Analyseverfahren der Konversationsanalyse (KA), der Interaktionalen Linguistik (IL) und der Multimodalen Analyse (MA) (vgl. u.a. auch Sidnell 2006; Selting 2013). Ich werde zeigen, wie Erzähler/innen die folgenden Arten von Ressourcen in designierten Praktiken der Konstruktion und 
Kontextualisierung von Höhepunkten von Belustigungs- und Beschwerdeerzählungen in ihren sequenziellen Kontexten verwenden:

- verbale Ressourcen aus dem Bereich der Rhetorik, Lexiko-Semantik, Syntax, Phonetik-Phonologie;

- vokale Ressourcen aus den Bereichen der Prosodie und Stimmqualität;

- visuelle oder sichtbare Ressourcen aus den Bereichen der Körperhaltung und ihrer Veränderung, Kopfbewegungen, Blickverhalten, Handbewegungen und Gesten.

Die Höhepunkte von Erzählungen und die Reaktionen darauf werden in der Regel nicht in einer neutralen Art und Weise präsentiert, sondern als emotional involviert, indem vor allem geeignete ,markierte oder ,saliente' Ressourcen für das verbale, vokale und visuelle Display kombiniert und gleichzeitig verwendet werden. Der ,markierte' oder ,saliente' Gebrauch eines Mittels ist dabei immer ein auffällig gemachter, ein auffälligerer Gebrauch im Vergleich zu seinem ,unmarkierten', ,unsalienten' und unauffälligen Gegenstück (vgl. auch Selting 1995a, 1996).

Der kontextsensitive Gebrauch ko-okkurrierender verbaler, vokaler und visueller Ressourcen in ihren sequenziellen Kontexten wird als holistische, ganzheitliche Praktik beschrieben, mit der der Höhepunkt der konversationellen Erzählung als Handlung innerhalb der Aktivität Erzählen erkennbar gemacht wird. Die Analyse wird validiert mit Hilfe der Analyse der Rezipientenreaktion. Aus Platzgründen kann ich nur jeweils ein einziges Beispiel für belustigende und für Beschwerdeerzählungen behandeln; in beiden Fällen reagiert die Rezipientin affiliativ.

\subsection{Der Höhepunkt einer Erzählung mit affiliativen Reaktionen}

Anhand der folgenden Beispielanalyse werde ich die Konstruktion von Höhepunkten von belustigenden Erzählungen mit voll affiliativen Reaktionen nachzeichnen. ${ }^{5}$

Vor dem Einsatz des Beispiels (1) hatte Sandra eine selbst-ironische Erzählung projiziert. Sie erzählt Emma, wie sie entgegen ihrer Prinzipien dazu kam, ihrer Tochter die Bravo zu kaufen: Nachdem sie sich zuvor geweigert hatte, ihrer Tochter diese Zeitschrift zu kaufen, kam sie eines Tages verspätet von der Arbeit nach Hause und fand einen Zettel ihrer Tochter vor: Hallo Mama, bin im Aldi. Sie suchte ihre Tochter dann im Aldi, wo diese eine halbe Stunde gesessen und die Bravo gelesen hatte.

5 Zu belustigenden Erzählungen vgl. auch Kotthoff (1995, 2006, 2007). 
(1)

045

048

049 Emm:

050 San:

052 Emm:

053

054 San:

055 San:

056

\section{LoE_VG_04_Bravo-Geschichten: Ausschnitt ab 17:19}

San: nÄchsten tAg komme ich zu spät von der

Arbeit nach HAUse, (.)

liegt n zETtel im |flur,

|((Augenbrauen hoch, | Zeigegeste)) |

(0.9) <all, mit downstep, präzise Artikulation>

| $\uparrow \uparrow h A l l o /$ mAma / bIn im /ALdi; >

| (( mit blankem "Pokerface"gesicht ) ) |

|((San \& Emma sehen sich an )) |

$(0.9)$

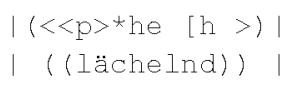




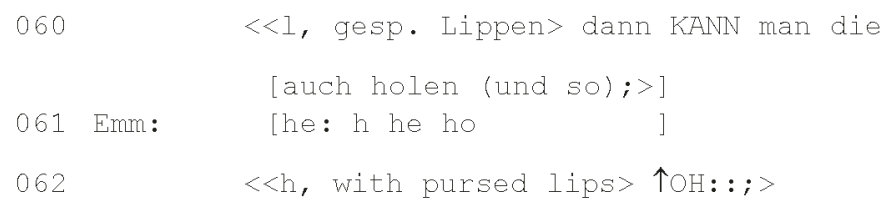

2. Teil:

affiliativ

Die sequenzielle Organisation von Sandras Erzählung und Emmas Reaktionen ist in Tabelle 1 dargestellt.

Tab. 1: Sequenzielle Organisation von Sandras Erzählung und Emmas Reaktionen

\begin{tabular}{lll}
\hline Segmente & Sandras Handlungen & Emmas Reaktionen \\
\hline $13-14$ & $\begin{array}{l}\text { Ankündigung mögl. Erzählung } \\
\text { (nicht gezeigt) }\end{array}$ & \\
\hline 45 & Einleitung des analysierten Ereignisses & \\
\hline $46-48$ & Dramatisierung & $\begin{array}{l}\text { minimal } \\
\text { = Fortsetzung abwartend? }\end{array}$ \\
\hline 50 & Fortsetzung & \\
\hline 51 & Höhepunkt (= 1. Teil) & Lachen + Bewertung (=2. Teil) \\
\hline $52-53$ & & \\
\hline 55 & Zustimmung mit Bewertung & Zustimmung + Lachen (=2. Teil) \\
\hline 56 & $\begin{array}{l}\text { Evaluation 1 }- \text { in Erzählwelt: }(=1 . \text { Teil) } \\
\text { Explikation der Pointe der Erzählung: } \\
\text { moralisches Resultat }\end{array}$ & \\
\hline $58-60$ & Evaluation 2 - im Hier und Jetzt $(=1$. Teil) & Lachen + Zustimmung (=2. Teil) \\
\hline
\end{tabular}

\subsubsection{Der Höhepunkt von Sandras Erzählung}

Nach der Einleitung und der Dramatisierung vorheriger Ereignisse in den Segmenten 45-48 präsentiert Sandra in Segment 51 den Höhepunkt ihrer Erzählung: da saß die seit ner <<höher + hoch bleibend $>/ \uparrow ! H A L !$ ben stunde im /Aldi drüben und hat /BRAvo ge[lesen;>. Diesen werde ich genauer untersuchen.

Die folgenden Ressourcen werden eingesetzt, um die Turnkonstruktionseinheit in Segment 51 als Höhepunkt der Erzählung erkennbar zu machen: 
- Rhetorisch und lexiko-semantisch stellt Sandra das eine halbe Stunde lange Sitzen und Bravo-Lesen ihrer Tochter im Supermarkt mit einer Extremformulierung dar (Pomerantz 1986).

- Syntaktisch konstruiert sie einen komplexen Satz, den sie mit dem topikalisierten Adverb da beginnt.

- Prosodisch realisiert sie einen Tonhöhensprung zu einem hohen Tonhöhengipfel in der extra starken Akzentsilbe der Phrase seit ner $\uparrow !$ HAL!ben stunde; die Tonhöhe im Rest der Intonationsphrase fällt kontinuierlich, bleibt aber bis zum Schluss relativ hoch. Die drei Akzentsilben in den Wörtern $\uparrow ! H A L ! b e n$, Aldi und BRAvo sind rhythmisch organisiert, d.h. die zweite und dritte Akzentsilbe folgen in perzeptiv ähnlichen Zeitabständen auf die jeweils vorherige Akzentsilbe, auch wenn die Akzenteinheiten hier relativ lang sind und aus jeweils fünf Silben bestehen.

Das PRAAT-Bild 1 (Abb. 1) zeigt die hohen Tonhöhengipfel im Segment 51; zum Vergleich zeigt das PRAAT-Bild 2 (Abb. 2) die viel niedrigeren Gipfel im späteren Segment 56.

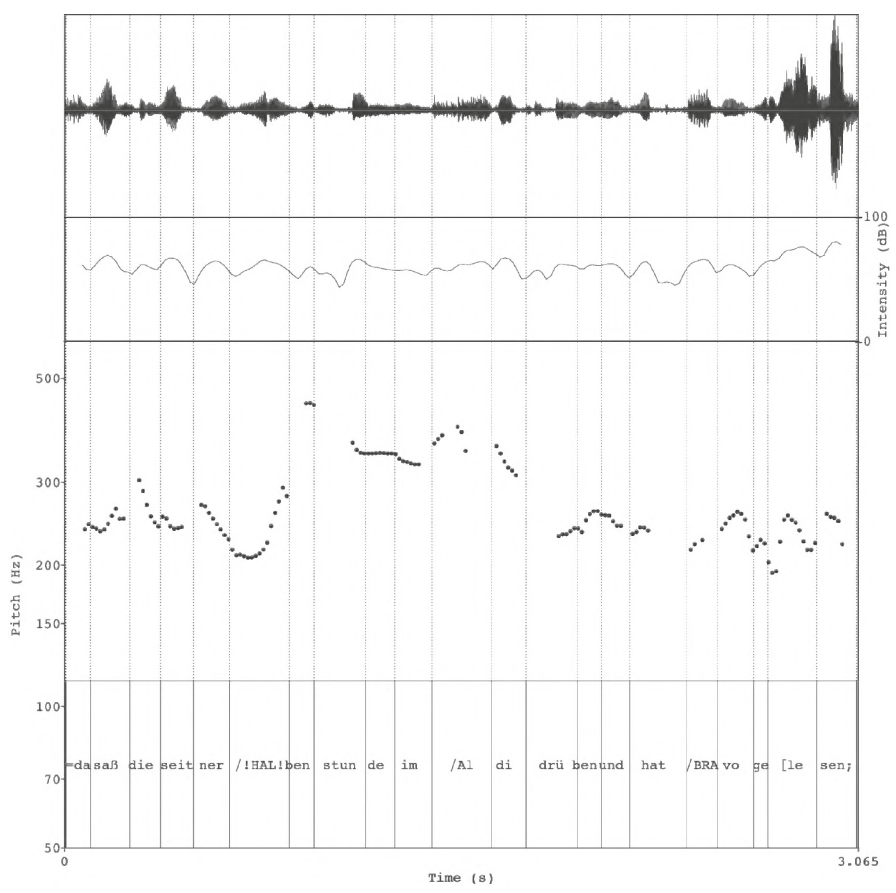

Abb. 1: PRAAT-Bild 1: Segment 51 


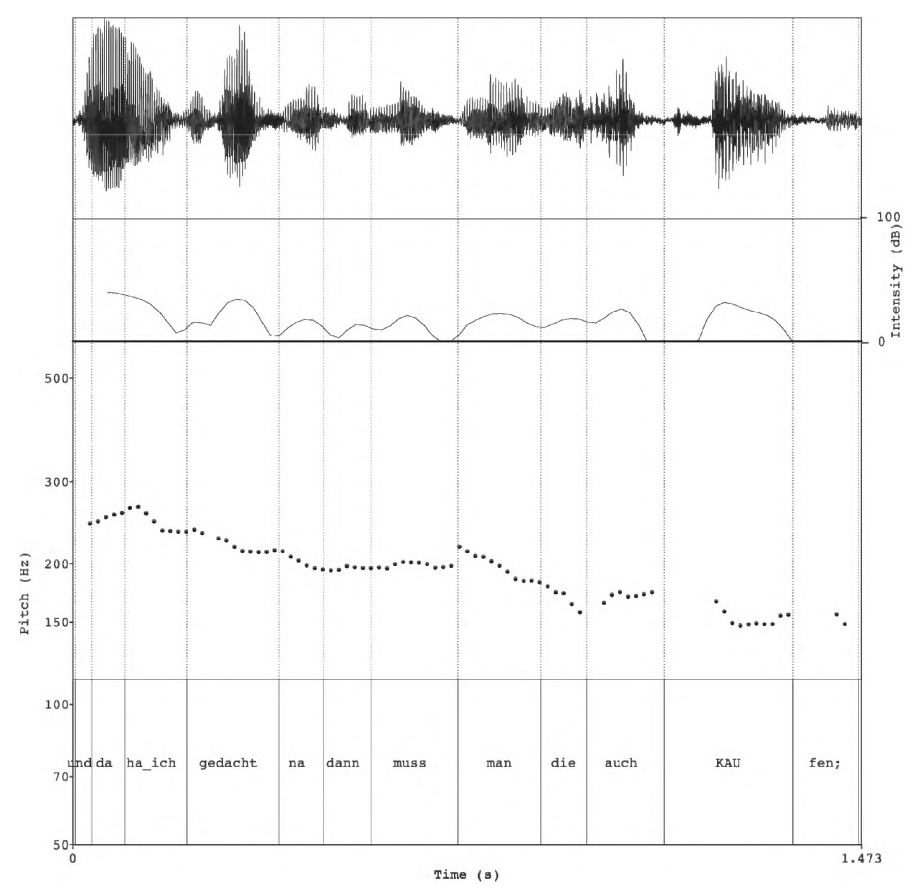

Abb. 2: PRAAT-Bild 2: Segment 56

Sichtbar hebt Sandra in Segment 51 ihren Arm und ihren Daumen noch höher als zuvor; sie zeigt immer noch nach hinten. Gleichzeitig mit den Wörtern $\uparrow$ !HAL!ben stunde hebt sie kurz ihre Augenbrauen, was man im Standbild 1 (Abb. 3) sehen kann. Alle drei Akzentsilben dieser Intonationsphrase werden von leichtem Kopfnicken begleitet. Sandras Gesicht zeigt einen amüsierten, lächelnden Ausdruck, bis sie am Ende der Phrase in ein lautloses Lachen ausbricht. Ihr Blick ist die ganze Zeit auf Emma gerichtet.

Die Analyse, dass Sandra die beschriebenen Ressourcen zur Kontextualisierung des Höhepunktes ihrer Erzählung einsetzt, wird durch den Einbezug von Emmas Reaktion gestützt: Nachdem Emma schon seit dem Segment 49 gelächelt hat, öffnet sich ihr Gesichtsausdruck im Verlauf des Segments 51 immer mehr. Dabei passt sie ihr zunehmendes Lächeln im Verlauf des Segments 51 genau an Sandras zunehmendes Lächeln an (vgl. auch Jefferson 1979). In Segment 52, gleichzeitig mit dem Ende von Sandras Segment, und unmittelbar nach dem Wort $B R A v o$, mit dem Sandra das relevanteste Ereignis ihrer Erzählung benennt, bricht Emma in Lachen aus. Das ist im Standbild 2 (Abb. 4) zu sehen. Emmas Lachen reagiert also unmittelbar auf Sandras Erkennbarmachen des Höhepunktes ihrer 
Erzählung mit Hilfe markierter lexikalischer, prosodischer und visueller EinzelRessourcen, die hier zusammen die ganzheitliche Praktik der Kontextualisierung des Höhepunktes der Erzählung ausmachen.

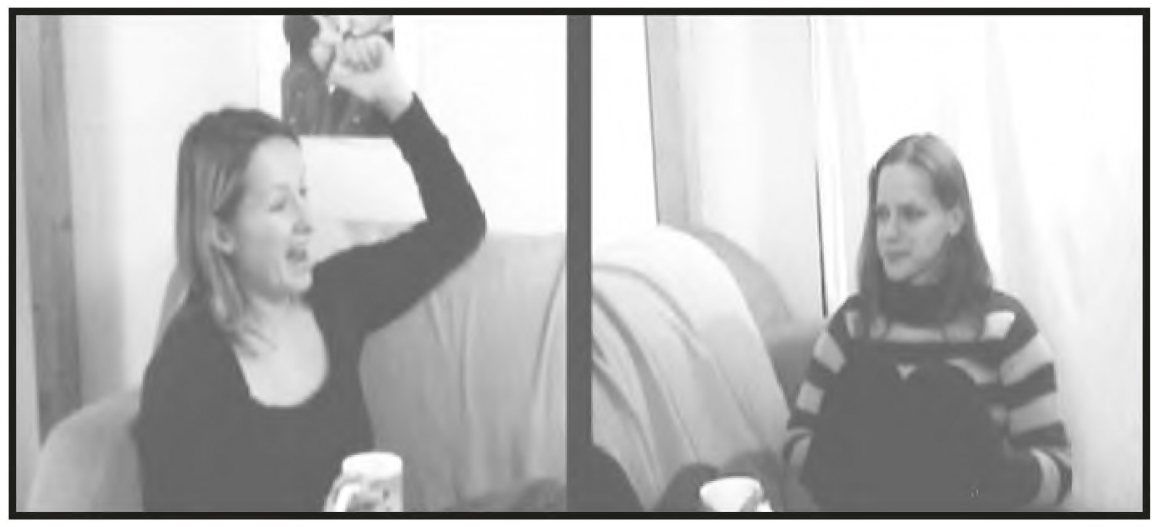

Abb. 3: Standbild 1: Sandras und Emmas Gesichtsausdrücke bei der Silbe !HAL! im Segment 51

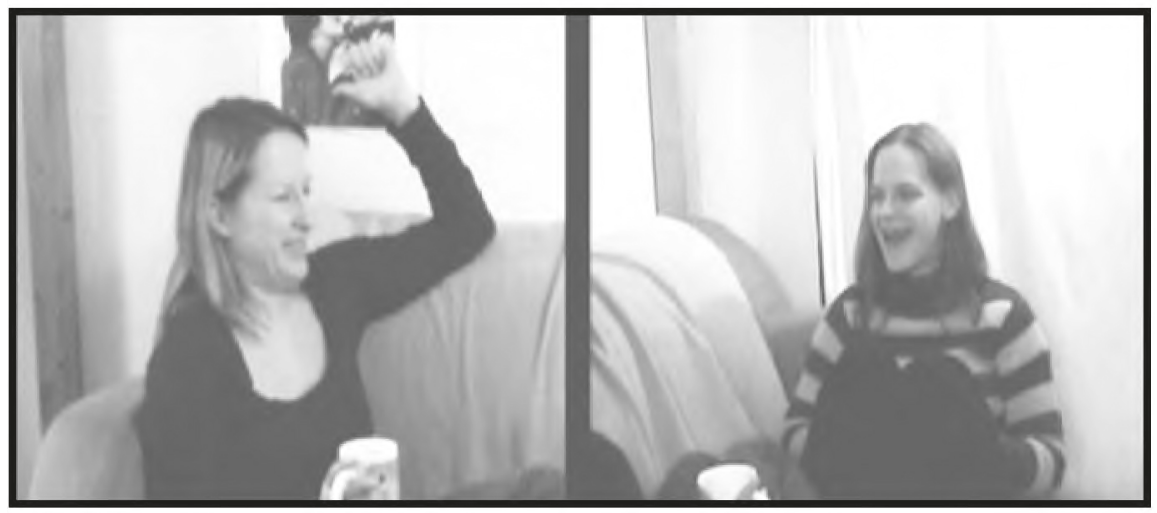

Abb. 4: Standbild 2: Sandras und Emmas Gesichtsausdrücke bei der letzten Silbe von Segment 51

Gleichzeitig mit Sandras Lachen, das in Segment 54 notiert ist, gibt Emma in den Segmenten 53-55 eine zustimmende positive Bewertung: <<hoch, lachend, mit gespitzten Lippen $>$ [个Oh dis_is] (aber) voll SÜSS; >=, der Sandra dann zustimmt: $=" J A: ;$. 
Emma formuliert nach dem Lautobjekt Oh (Reber 2012) eine verbal explizite Bewertung mit einem gesteigerten lexikalischen Ausdruck: voll SÜSS. Prosodisch wird diese mit hoher Tonhöhe, lachend und mit gespitzten Lippen formuliert, so dass sie wie „motherese“ oder „baby-talk“ klingt. ${ }^{6}$ Sowohl ihr Lachen in Zeile 52 als auch ihre Bewertung in Zeile 53 sind Evidenz dafür, dass Emma Sandras vorherige Turnkonstruktionseinheit in Segment 51 als den Höhepunkt ihrer belustigenden Erzählung versteht und mit-konstruiert. Emmas Reaktion ist eindeutig affiliativ. Sandra stimmt Emmas Bewertung zu und schließt damit in Segment 55 diese Sequenz.

\subsubsection{Sandras Expansionen}

Im weiteren Verlauf der Sequenz, in den Segmenten 56-62, formuliert Sandra den Relevanzpunkt ihrer Erzählung, den Grund, weshalb sie sie erzählt hat: Obwohl sie ihrer Tochter die Bravo nicht kaufen will und es nicht mag, dass ihre Tochter sie liest, hat sie doch der Tochter nachgegeben.

Rhetorisch wechselt Sandra zwischen den Segmenten 56 und 58-60 die Perspektive von der Darstellung der rekonstruierten Affekte in der Erzählwelt ins Hier und Jetzt der Erzählsituation. In den Segmenten 56-60 rechtfertigt sie ihren Kauf der Bravo für ihre Tochter also zweimal: zuerst als Teil der Erzählung (Segment 56), dann als Reformulierung im Hier und Jetzt (Segment 58-60).

Prosodisch artikuliert Sandra ihre Rechtfertigungen - wie zuvor Emma ihre Bewertung - mit gespitzten Lippen, die auch diese wie „motherese“ oder „babytalk“ klingen lassen. Und auch Emma bleibt in ihren Reaktionen bei dieser Artikulationsweise. Beide Gesprächsteilnehmerinnen legen damit nahe, dass sie Sandras Verstoß gegen ihre eigenen Prinzipien humorvoll sehen. Dennoch wird prosodisch ein klarer Kontrast hergestellt: Während die Erzählerin Sandra mit tiefem Tonhöhenregister Belustigung und gespielte Zerknirschtheit darstellt, legt die Zuhörerin Emma mit hohem Tonhöhenregister nur Belustigung nahe.

Dieses Beispiel hat gezeigt, wie die Erzählerin Sandra im Kontext ihrer angekündigten Erzählung nach einer Dramatisierung die gleichzeitige Verwendung der beschriebenen Arten von Ressourcen in diesem sequenziellen Kontext als Praktik verwendet, um den Höhepunkt ihrer belustigenden Erzählungen erkennbar zu machen, sodass die Rezipientin diesen erkennen und angemessen reagieren kann. Die Rezipientin reagiert mit Lachen, so wie es von der Erzählerin auf

6 Dies entspricht den prosodisch unmarkierten Verwendungsweisen von süß, die Szczepek Reed (2014, S. 166 ff.) beschreibt. 
verschiedene Weise relevant gemacht wurde. Danach lachen oder lächeln beide, und sie lachen zusammen. Die Verdeutlichung des Punktes der belustigenden Erzählung in der Erzählwelt wird ergänzt durch eine Reformulierung im Hier und Jetzt der Erzählsituation. Auf diese Weise wird die hergestellte Freude oder Belustigung von beiden Gesprächsteilnehmerinnen noch etwas länger ausgekostet.

\subsection{Der Höhepunkt einer Beschwerdeerzählung mit affiliativen Reaktionen}

Auch im hier gezeigten Beispiel einer Beschwerdeerzählung zeigt der Rezipient affiliative Reaktionen. ${ }^{7}$

Im Beispiel (2) liefert Carina eine Beschwerdeerzählung darüber, wie sie nach nur fünf Minuten versehentlichen Parkens auf einem Behindertenparkplatz einen Strafzettel erhalten hat. Hajo reagiert voll affiliativ.

(2) LoE_VG_03_Parkausweis Gehbehinderte

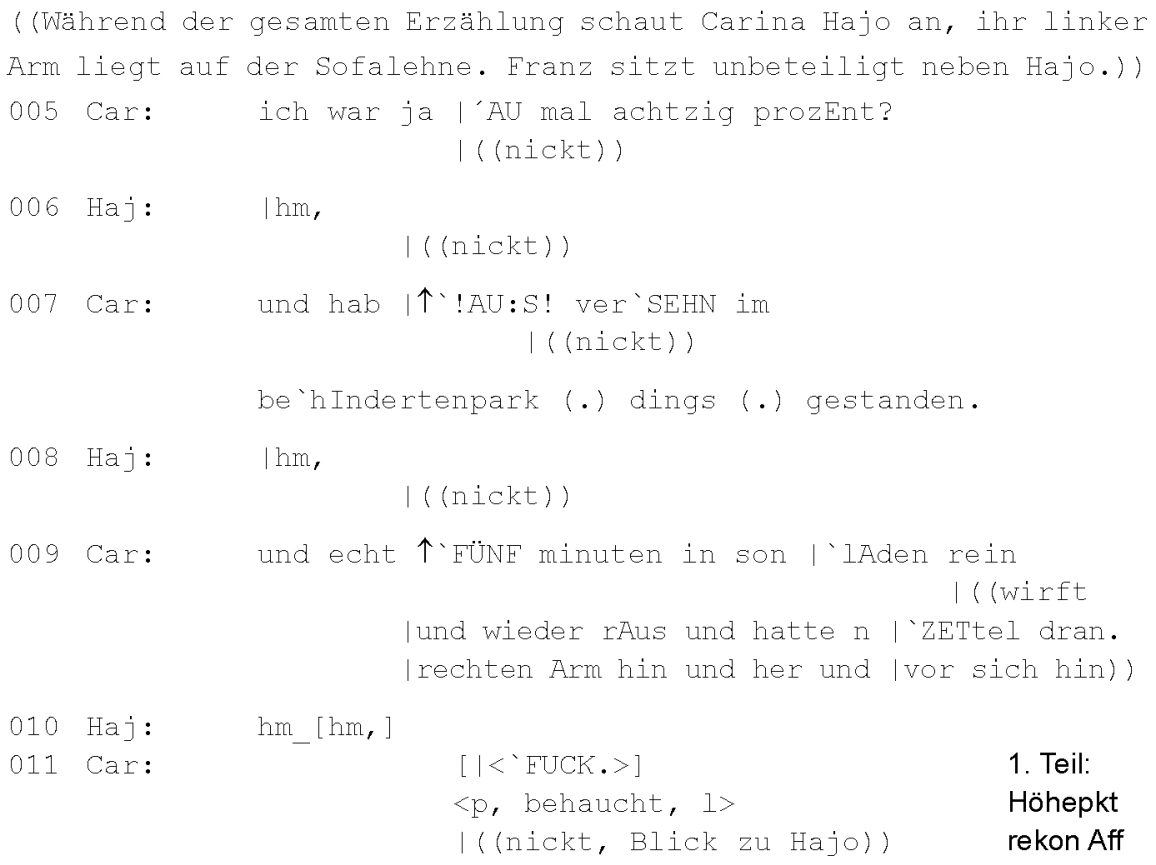

7 Zu Beschwerdeerzählungen bzw. complaint stories vgl. auch Drew/Holt (1988), Drew (1998), Christmann/Günthner (1996), Günthner (2000), Selting (2010, 2012). 


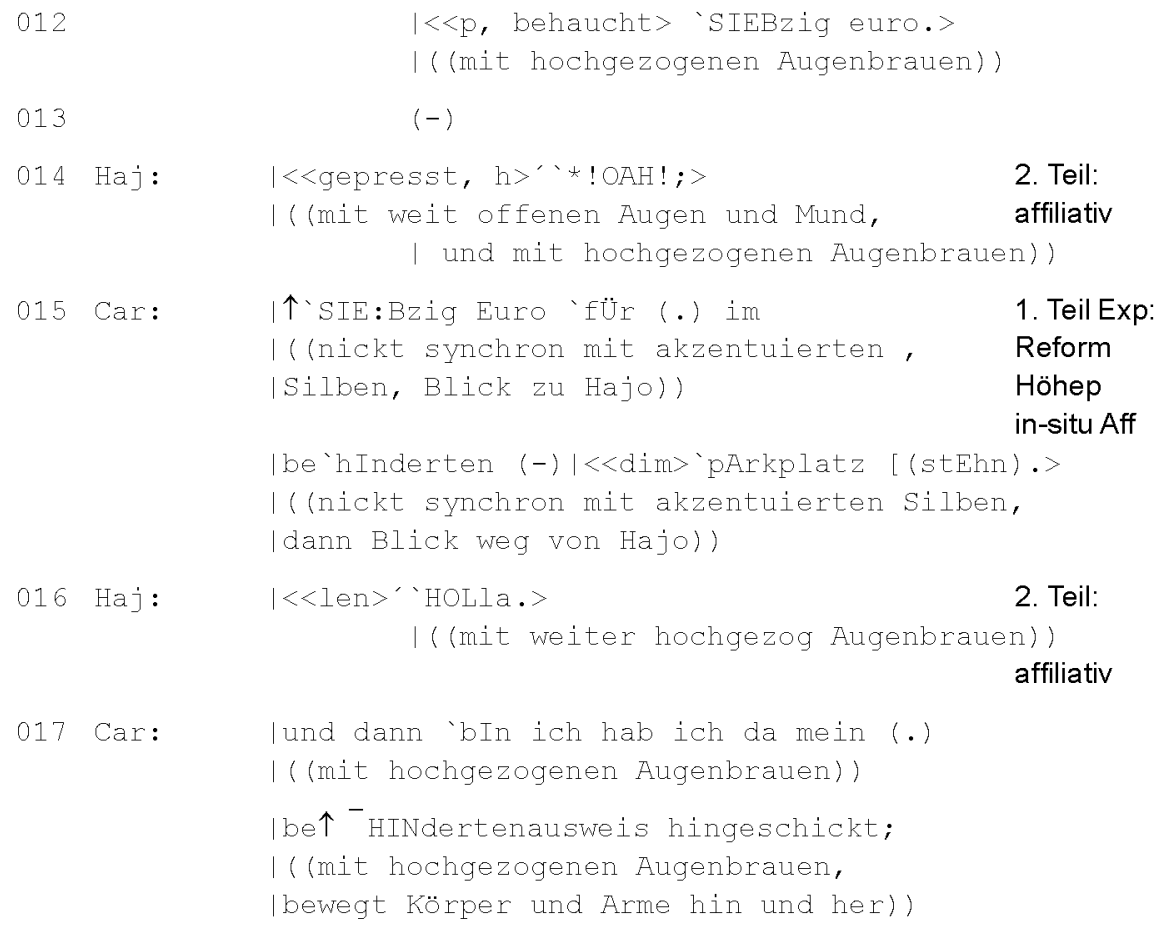

Die sequenzielle Entwicklung dieser Sequenz ist in Tabelle 2 zusammengestellt.

Tab. 2: Sequenzielle Organisation von Carinas Beschwerdeerzählung und Hajos Reaktionen

\begin{tabular}{lll}
\hline Segmente & Carinas Handlungen & Hajos Reaktionen \\
\hline 05 & Ankündigung der Erzählung & \\
\hline 06 & & Ratifizierung \\
\hline $07-22$ & Erzählung mit: & Rezeptionssignale \\
\hline $07-10$ & Ereigniskette & \\
\hline $11-12$ & $\begin{array}{l}\text { Höhepunkt: } \\
\text { Beschwerdeereignis }\end{array}$ & $\begin{array}{l}\text { affiliative Reaktionen: } \\
\text { „Sound object“, response cry }\end{array}$ \\
\hline 14 & & \\
\hline 15 & $\begin{array}{l}\text { Expansion/Reformulierung } \\
\text { des Höhepunktes }\end{array}$ \\
\hline
\end{tabular}




\begin{tabular}{lll}
\hline Segmente & Carinas Handlungen & Hajos Reaktionen \\
\hline 16 & $\begin{array}{l}\text { weitere affiliative Reaktion: } \\
\text { response cry }\end{array}$ \\
\hline $17 \mathrm{ff}$. & Fortsetzung der Erzählung & \\
\hline
\end{tabular}

Wie macht Carina den Höhepunkt ihrer Erzählung erkennbar?

\subsubsection{Der Höhepunkt von Carinas Erzählung}

In Überlappung mit Hajos Rezeptionssignal in Segment 10 produziert Carina in Segment 11 das englische Schimpfwort FUCK. und nennt dann in Segment 12 die Summe, die sie als Strafe zahlen musste: 'SIEBzig euro.

Rhetorisch und lexiko-semantisch fällt Carinas Schimpfwort in Segment 11 natürlich auf. Außerdem realisiert sie damit einen Code-Switch ins Englische. Diese Turnkonstruktionseinheit präsentiert das Knöllchen als ein sehr negativ bewertetes Ärgernis. Für die jungen Leute ist die Höhe der Summe, die in Segment 12 angegeben wird, 70 Euro, eine Extremformulierung. Syntaktisch wird der Höhepunkt mit minimal kurzen, dichten Konstruktionen mit ein oder zwei Wörtern realisiert (vgl. Günthner 2006). Prosodisch realisieren die Einheiten fallende Akzenttonhöhenverläufe und final fallende Tonhöhe. Mit ihrer Stimmqualität stellt Carina jedoch einen Kontrast zu ihren vorherigen Einheiten her: FUCK wird in einem tieferen Tonhöhenregister produziert, sowohl FUCK als auch SIEBzig euro werden leise und mit behauchter Stimme realisiert. Außerdem produziert Carina das Schimpfwort FUCK mit einem gleichzeitigen Kopfnicken und zieht bei den Wörtern SIEBzig euro die Augenbrauen hoch. Ihr Gesichtsausdruck beim Segment 12 ist im Standbild 3a (Abb. 5) zu sehn.

Carina kombiniert alle diese Ressourcen, um die Einheiten in den Segmenten 11 und 12 als emotional beteiligten Höhepunkt ihrer Erzählung erkennbar zu machen. Die starken negativen Bewertungen legen die Interpretation des spezifischen Affekts als, Ärger‘ oder,Entrüstung‘ nahe, weil sie sich unfair behandelt sieht (vgl. Günthner 2000). Dieser Affekt wird als rekonstruierter Affekt dargestellt, den sie in ihrer Erzählwelt erlebte. Im Vergleich zu anderen Beschwerdeerzählungen, in denen die rekonstruierten Affekte, ̈̈rger‘ oder ,Entrüstung mit lauteren Mitteln dargestellt werden, stellt Carina ihren Ärger hier mit eher leisen Mitteln dar: Leise, mit behauchter Stimme und tiefem Tonhöhenregister. Damit stellt sie ihren Affekt offenbar als eine vergangene Erfahrung dar, mit der sie sich nun abgefunden hat. 


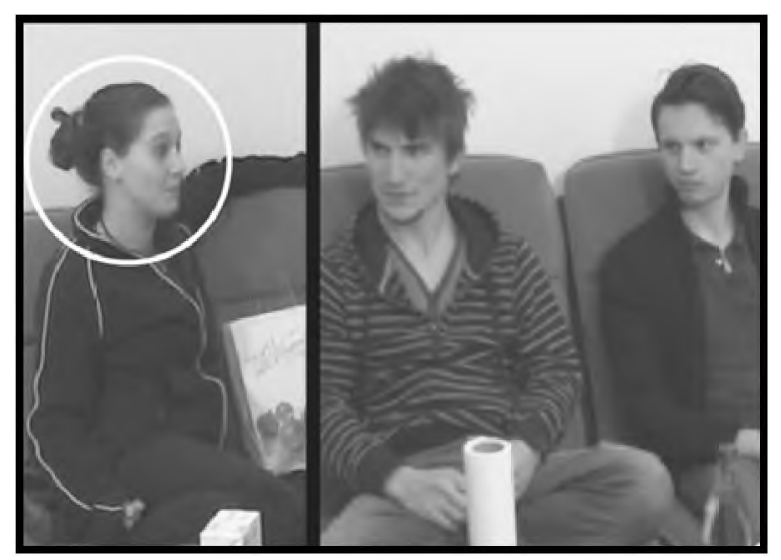

Abb. 5: Standbild 3a: Segment 12

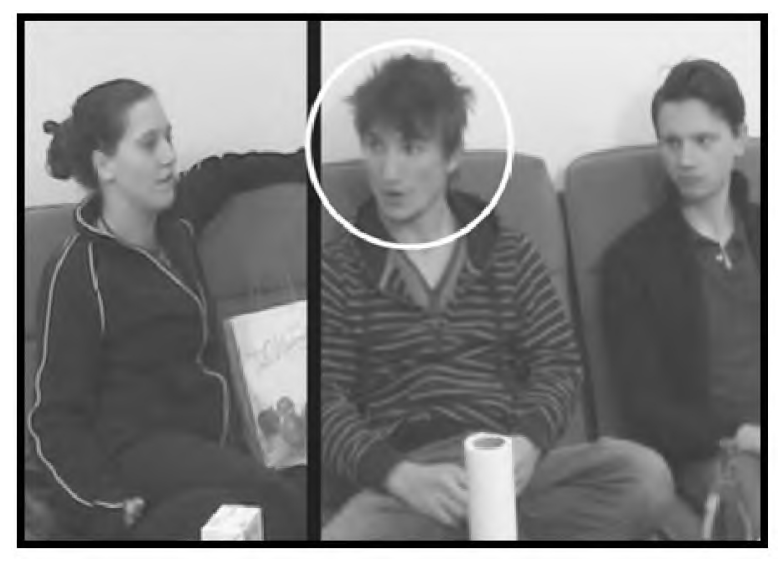

Abb. 6: Standbild 3b: Segment 14

Die Analyse des gezeigten Affekts als ,Ärger oder ,Entrüstung ${ }^{`}$ kann mit der Einbeziehung der Reaktion des Rezipienten Hajo validiert werden. Nach einer kurzen Pause reagiert er im Segment 14 mit dem Lautobjekt (Reber 2012) “*!OAH!; in einem hohen Tonhöhenregister, mit steigend-fallender Tonhöhenbewegung, und mit einer gespannten, gepressten Stimmqualität. Zugleich sieht er Carina mit plötzlich weit aufgerissenen Augen, offenem Mund und hochgezogenen Augenbrauen an. Das ist im Standbild 3b (Abb. 6) zu sehen. 
Alle diese Ressourcen zusammen realisieren einen konventionellen response cry (Goffman 1978), einen „Reaktionsschrei“ zur Signalisierung von Überraschung und affiliativer Übereinstimmung mit dem von Carina dargestellten Affekt. Hajos hochgezogene Augenbrauen im Segment 14 (wie auch später in 16) sind genau an Carinas hochgezogene Augenbrauen in 12 angepasst. Seine Pause im Segment 13 und seine etwas verspätete Reaktion kann dabei als weiteres Zeichen seiner Überraschung analysiert werden (vgl. auch Wilkinson/Kitzinger 2006).

\subsubsection{Carinas Expansion ihres Höhepunktes}

Im Segment 15 expandiert Carina ihren Höhepunkt: $\uparrow$ 'SIE:Bzig Euro 'für (.) im be ' hInderten $(-) /<<$ dim $>$ 'pArkplatz $\left[(\right.$ stEhn $){ }^{8}$

In diesem Fall ist es nicht nur die verbale und non-verbale Markierung oder Salienz der Äußerung, die Carinas Affekt darstellt, sondern auch die Tatsache, dass sie den ärgerlichen Sachverhalt erneut formuliert, in ähnlichen Worten wie zuvor. Sie zieht die Aufmerksamkeit noch einmal auf diese ärgerliche Sache und schafft damit eine neue Gelegenheit für Hajos Reaktion. Dennoch erscheint Carinas zweite Formulierung des Höhepunktes schwächer und, ruhiger` als die erste.

Auch diese Analyse kann wieder durch den Einbezug von Hajos Reaktion im Segment 16, “HOLla., validiert werden. Im Vergleich zu seiner vorherigen Reaktion im Segment 14 ist dieser zweite „Reaktionsschrei“ jedoch prosodisch und non-verbal weniger markiert. Prosodisch wird er in langsamem Tempo realisiert, mit markierter steigend-fallender Tonhöhenbewegung, die Stimme klingt nicht mehr gepresst. Seinen früheren Gesichtsausdruck behält er jedoch bei: Er schaut weiterhin Carina mit weit aufgerissenen Augen und hochgezogenen Augenbrauen an. Das heißt: Genau so wie Carinas zweite Formulierung des Höhepunkts ihrer Erzählung schwächer als die erste war, genau so ist nun Hajos zweiter „Reaktionsschrei“ schwächer als sein erster.

Die Grundfrequenz von Hajos Reaktionen ist in den PRAAT-Bildern 3-5 (Abb. 7-9) zu sehen:

8 Auf die Details kann ich hier nicht eingehen (siehe dazu Selting 2010). 


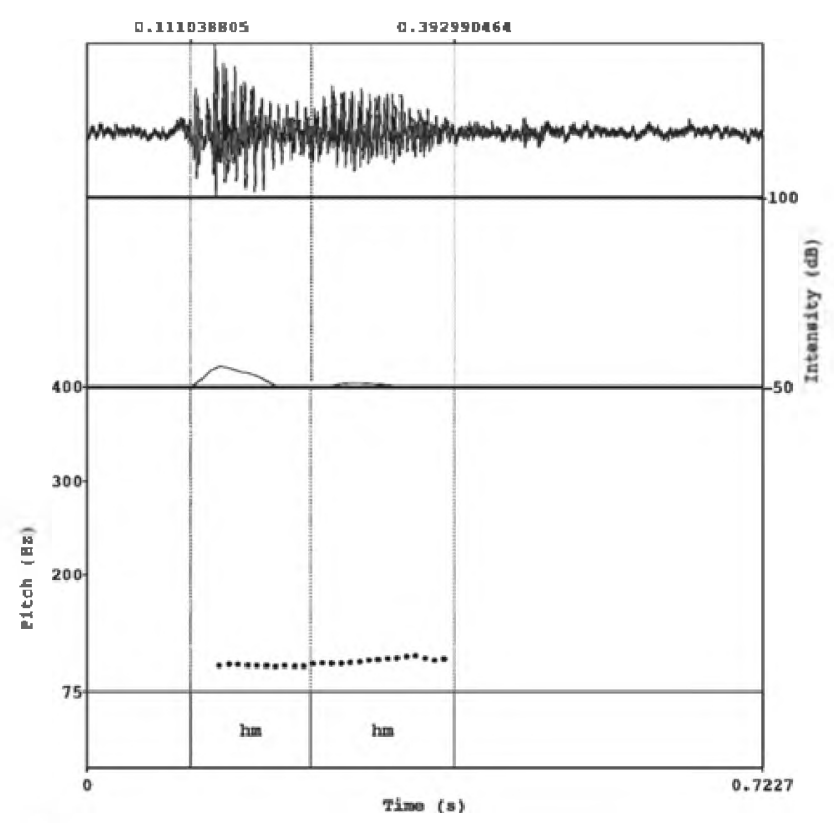

Abb. 7: PRAAT-Bild 3: Segment 10

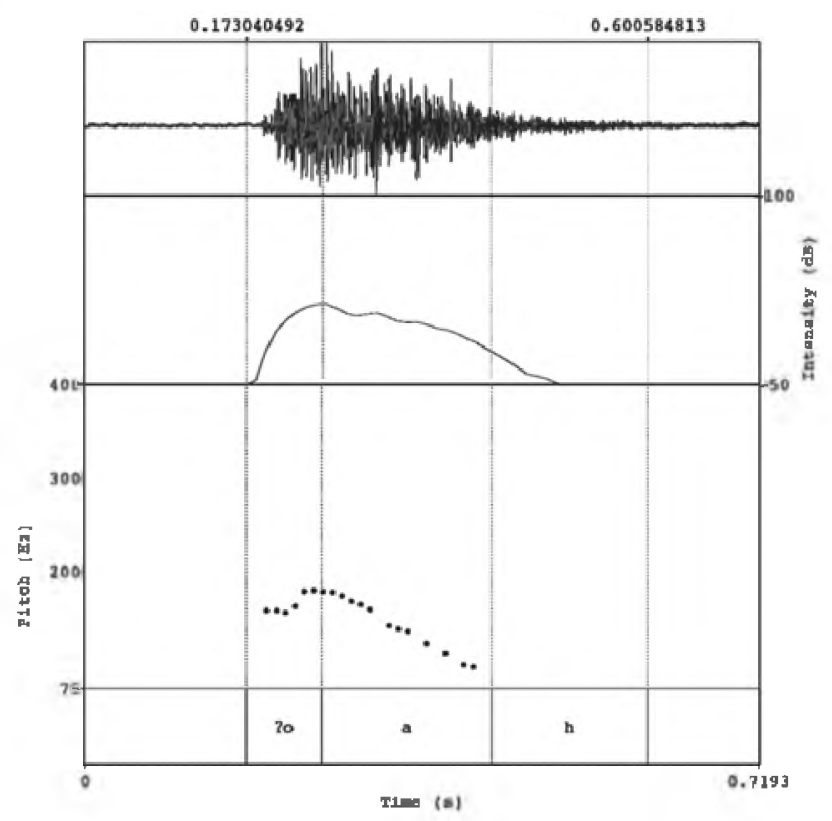

Abb. 8: PRAAT-Bild 4: Segment 14 


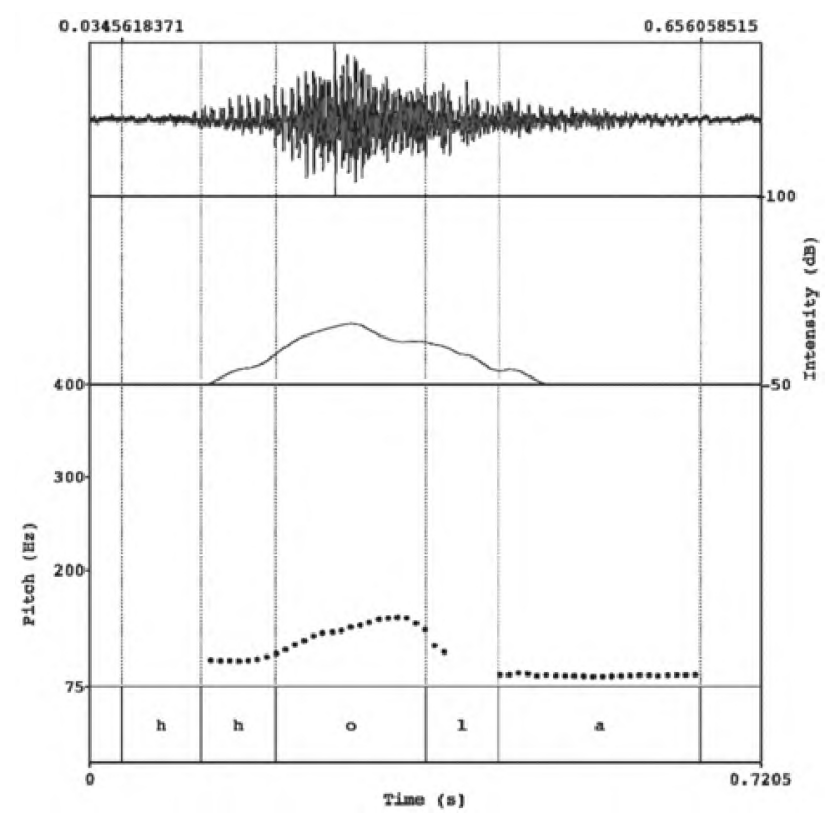

Abb. 9: PRAAT-Bild 5: Segment 16

Während Abbildung 7 die unmarkierte Grundfrequenz des einfachen Rezeptionssignals $h m \_h m$ in Segment 10 zeigt, zeigt Abbildung 8 das wesentlich bewegtere und höher steigend-fallende *!OAH!; in Segment 14, und Abbildung 9 das schon wieder weniger hoch bewegte steigend-fallende “ HOLla. in Segment 16.

Hajo passt seine Reaktionen also ganz genau an Carinas jeweils vorherige Darstellung an und nimmt damit Carinas Beschwerdeerzählung voll affiliativ auf.

Dieses Beispiel hat gezeigt, dass bei einer Beschwerdeerzählung eine Erzählerin ein Ereignis als ärgerlich darstellt und dabei verbale, vokale und visuelle Ressourcen einsetzt, um ihren Affekt erkennbar zu machen. Vom Rezipienten wurden Lautobjekte als affiliative „Reaktionsschreie“ verwendet. Nach ausreichend affiliativen Reaktionen des Rezipienten werden die Affektdisplays der Teilnehmer in der Expansion des Höhepunktes schwächer. 


\section{Fazit}

Zwischen der Handlung der Herstellung und Kontextualisierung des emotional beteiligten Höhepunktes einer Erzählung und der Praktik des Einsatzes von verbalen, vokalen und visuellen Ressourcen besteht eine indexikalische und reflexive Relation, die ich mit Bezug auf Schegloffs (2000) Granularitätsebenen darstellen möchte. Ich werde bei der Darstellung der Ergebnisse meiner Analysen von der kleineren zur größeren Auflösung, d.h. ausgehend von der Handlung über die Praktik zu den detaillierteren Ressourcen fortschreiten.

\section{i. Auf der Granularitätsebene der Aktivitäts- und Handlungskonstitution}

Begrifflich ist die Erzählung als Ganze als Aktivität zu beschreiben, für deren geordnete Gestaltung mehrere Handlungen nacheinander durchzuführen sind, u.a. Ankündigen der Erzählung, Elaborieren oder Dramatisieren der Ereignisse, und oft auch das Erkennbarmachen des Höhepunktes der Erzählung, was jeweils passende Reaktionen der Rezipienten relevant macht, mit denen sie die Erzählung ko-konstruieren.

Erzählungen werden meist mit erhöhter emotionaler Beteiligung dargeboten. Die konkreten Affekte, die die Erzähler erkennbar machen, werden vor allem an zwei Stellen deutlich gemacht: Zum einen wird in der Ankündigung der Erzählung auch die Art der Erzählung als lustige oder ärgerliche o.ä. verdeutlicht, zum anderen wird der Affekt bei der Präsentation von Höhepunkten der Erzählung dargestellt. In meiner Analyse hier habe ich Höhepunkte von Belustigungs- und Beschwerdeerzählungen untersucht.

\section{ii. Auf der Granularitätsebene der sequenziellen Organisation von Höhe- punkten von Erzählungen mit emotionaler Beteiligung}

Nach vorheriger Ankündigung und Dramatisierung werden Höhepunkte konversationeller Erzählungen mit erhöhter emotionaler Beteiligung erkennbar gemacht, die mithilfe der im Folgenden aufgeführten Praktiken multimodal dargeboten werden und eine passende Reaktion der Rezipienten relevant machen.

Sequenziell werden Höhepunkte mit erhöhter emotionaler Beteiligung in wenigstens zwei aufeinander folgenden Einzelsequenzen organisiert die zusammen eine Affekt-Darstellungs-Sequenz konstituieren. Im normativ erwartbaren ,Normalfall“" sind das: 


\section{Zwei Sequenzen für die gemeinsame Herstellung und Behandlung von Höhepunkten konversationeller Erzählungen mit emotionaler Beteiligung, bei affiliativen Reaktionen der Rezipienten}

Das Erkennbarmachen des Höhepunktes durch die Erzählerin macht i.d.R. eine affiliative Reaktion der Rezipienten relevant, woraufhin beide dann zur nächsten Sequenz fortschreiten können. Nur diesen Verlauf habe ich genauer gezeigt. Die beiden Sequenzen bestehen aus folgenden Einzelhandlungen:

\section{Erste Sequenz -}

\section{für Darbietung und Herstellung gemeinsamer Affektivität}

1. Teil Erzähler/in Produktion des/eines Höhepunktes der Erzählung mit Darstellung rekonstruierter Affektivität

2. Teil Rezipient/in affiliative Reaktion

Wenn die Rezipient/innen den 2. Teil der ersten Sequenz wie relevant durchführen, wird wenigstens eine weitere Nachfolgesequenz angeschlossen:

\section{Folge-Sequenz (kann mehr als einmal durchgeführt werden) - Expansion für Konsolidierung und Auskosten, und/oder Ausstieg aus, gemeinsamer Affektivität}

1. Teil Erzähler/in rekonstruierte und/oder in-situ Evaluation, Bestätigung, o. Ä., impliziert Aufnahme der Affiliation des Rezipienten

2. Teil Rezipient/in affiliative Reaktion

Im Beispiel (1) wurde die Folge-Sequenz zweimal durchgeführt. Im Beispiel (2) wurde sie einmal durchgeführt. Nach affiliativen Reaktionen der Rezipienten auf den ersten Teil der ersten Sequenz kann der Affektdisplay in der Folge-Sequenz schwächer ausfallen.

Die Kontextualisierung von Höhepunkten konversationeller Erzählungen mit Affektdisplay macht also Reaktionen der Rezipienten relevant. Affiliative Reaktionen werden bevorzugt.

Bei nicht-affiliativen Reaktionen der Rezipienten, wofür ich aber aus Platzgründen keine Beispiele zeigen kann, tritt demgegenüber ein anderer Verlauf der Sequenz ein: Nach einer nicht-affiliativen Reaktion des Rezipienten auf die Produktion des/eines Höhepunktes der Erzählung mit Darstellung rekonstruierter Affektivität reagiert die Erzählerin i.d.R. mit zunächst einer ersten und dann ggf. weiteren Expansionen, mit denen sie affiliative Reaktionen weiterhin relevant macht bzw. einfordert: z.B. mit weiteren Höhepunkten oder Evaluationen, weite- 
ren Darstellungen rekonstruierter oder in-situ Affektivität, Erläuterungen, Detaillierungen o.̈. Wenn Rezipienten auch nach diesen Expansionen und/oder Einforderungen keine affiliativen Reaktionen zeigen, zieht schließlich die Erzählerin zurück, indem sie z.B. aufgibt oder ihren Punkt ändert o. A.

Affiliative und nicht-affiliative Reaktionen der Rezipienten auf Höhepunkte von Erzählungen führen also zu sequenziell unterschiedlichen weiteren Interaktionsverläufen. Affiliative Reaktionen werden als präferierte und nicht-affiliative Reaktionen als nicht-präferierte behandelt.

Die Folge-Sequenzen der Affekt-Darstellungs-Sequenz sehen bei Belustigungsund Beschwerdeerzählungen tendenziell unterschiedlich aus:

- Höhepunkte mit Freude oder Belustigung werden oft noch etwas ausgekostet, von beiden Teilnehmern noch etwas "gefeiert". Hier sind die Affektdisplays der Erzähler und der Rezipienten in ihren affiliativen Reaktionen ähnlich, d.h. auf Belustigung wird mit Belustigung reagiert, auf Lachen mit Lachen, usw. (vgl. Selting i.Vorb.).

- In Höhepunkten von Beschwerdeerzählungen werden hingegen in unserem Korpus die Affektdisplays häufig auch vom Rezipienten abgemildert. Hier sind die Reaktionen der Rezipienten dann nicht ähnlich, sondern eher passend oder angemessen, d.h. auf Ärger und Entrüstung wird ggf. mit Beruhigung reagiert, als ob Rezipienten den Affekt de-eskalieren wollten, anstatt ihn durch Mit-Entrüstung weiter anzustacheln (vgl. Selting 2010). ${ }^{9}$

\section{iii. Auf der Granularitätsebene der Praktiken mit ihren verbalen, vokalen und visuellen Ressourcen}

Um die Affekt-Darstellungs-Sequenz herzustellen und um Höhepunkte mit emotionaler Beteiligung erkennbar zu machen, verwenden Erzähler/innen innerhalb dieser Sequenzen typischerweise einige der verbalen, vokalen und visuellen Ressourcen, die im Folgenden aufgelistet sind, in Kookkurrenz:

- Rhetorische Formate und Formen:

- als belustigend dargestellte Äußerungen oder Wörter in Rede- oder Gedankenwiedergabe,

- response cries,

- lakonisch kurze Formulierungen,

- $\quad$ erkennbares Einnehmen und Wechseln von Perspektiven usw.

9 Günthner (2000) fand dagegen eher Ko-Entrüstung der Rezipienten. Der Unterschied könnte auf das unterschiedliche Datenmaterial zurückzuführen sein. 
- Lexiko-semantische Formate und Formen:

- Extremformulierungen,

- lexikalische Einheiten mit impliziten zustimmungsfähigen Bewertungen,

- response cries usw.

- Syntaktische Formate und Formen:

- historisches Präsens,

- dichte Konstruktionen,

- kurze und prägnante Phrasen,

- aber auch komplexe Sätze,

- Topikalisierungen usw.

- Prosodische Formate und Formen:

- extra starke Akzente,

- dichte Akzentuierung,

- rhythmische Organisation der Akzentsilben,

- hohe Tonhöhengipfel,

- Veränderungen des Tonhöhenregisters,

- Tempo- und Lautstärkeveränderungen,

- Konstruktion von mehreren kurzen statt einer längeren Intonationseinheit usw.

- Stimmqualitäten:

- Lachpartikel innerhalb von Wörtern und verbalen Einheiten und/oder

- freistehendes Lachen,

- fingiertes oder gekünsteltes Lachen,

- lächelnde Stimme,

- Veränderungen der Stimmqualität (wie z.B. von oder zu: gespannter und hyperartikulierter Stimmqualität, präziserer Artikulation als in umliegenden Segmenten, Artikulation mit gespitzten Lippen, behauchter Stimme),

- auffälliges Räuspern usw.

- Sichtbare Ressourcen:

- lächelnder oder lachender Gesichtsausdruck,

- Veränderungen des Gesichtsausdrucks, z.B. zu oder von auffällig leer, ausdruckslos, belebt,

- gehobene Augenbrauen, Stirnrunzeln, weit geöffnete Augen,

- Kopfnicken,

- Blickveränderungen zwecks z.B. Fokussierung oder Dramatisierung,

- Zeigegesten usw.

Wie die Liste der einfachen Formen und komplexeren Formate zeigt, sind die Ressourcen, die in den Praktiken zur Kontextualisierung von Höhepunkten eingesetzt werden, vielfältig und recht unterschiedlich. Es gibt entgegengesetzte For- 
men, wie sowohl hohes als auch tiefes Tonhöhenregister. Keine der Ressourcen hat eine festgelegte interaktionale Bedeutung. Es sind auch nicht die einzelnen Formen oder Formate, die Höhepunkte und die beteiligten Affekte als solche erkennbar machen, sondern vielmehr deren Gebrauch in Praktiken. Die Ressourcen werden zu Ressourcenbündeln kombiniert und eingesetzt für Praktiken wie die Herstellung von Kontrast und Markiertheit oder Salienz, um Turnkonstruktionseinheiten im Vergleich zu vorhergegangenen Einheiten auffällig zu machen. Diese müssen dann im lokalen sequenziellen Kontext interpretiert werden.

Dennoch gibt es auch spezifische Ressourcen und Ressourcenbündel, die für die Kontextualisierung von Höhepunkten mit spezifischen Affekten eingesetzt werden:

Für belustigende Erzählungen sind das z.B.:

die Präsentation einer Entität als zustimmungsfähig durch den Gebrauch von lexikalischen Einheiten und Bewertungen, die Zustimmung nahelegen, und Lächeln und Lachen.

Für Beschwerdeerzählungen sind das z.B.:

- die Präsentation des Beschwerdeobjekts als „schrecklich“ (vgl. ,egregious“ bei Drew 1998, S. 322) mithilfe von Schimpfwörtern, negativen Bewertungen,

- gespannte oder gepresste Stimmqualität,

- Gesichtsausdrücke mit Anstarren und Stirnrunzeln.

Die Interpretation dieser Praktiken und Ressourcen ist jedoch immer kontext- und sequenzabhängig. ${ }^{10}$

Und um zum Schluss noch einmal auf das Thema des Bandes zurückzukommen: Muss es so kompliziert sein? Warum und wozu wird der Begriff der Praktik tatsächlich gebraucht?

Der Begriff ,Praktik‘ deckt ungefähr das ab, was z.B. in der Gumperzschen Kontextualisierungstheorie (Gumperz 1982) als ,Kontextualisierungsverfahren' bezeichnet wird, ,Ressourcen' entsprechen den ,Kontextualisierungshinweisen‘. Gegenüber dem Begriff ,Kontextualisierungshinweis‘ betont der Begriff ,Ressource' stärker und begrifflich offensichtlicher, dass diese Mittel und Signale von Sprechern je nach Bedarf gewählt und verwendet werden können, aber nicht

10 Dies exemplifiziert, was Schegloff (1996, S. 56) als „the central prospect“ einer grammar in interaction ansieht, nämlich die Beschreibung ,that grammar stands in a reflexive relationship to the organization of a spate of talk as a turn", und was er - allerdings mit Bezug auf andere Phänomene - konzipiert als „positionally sensitive grammars“, oder „positionally specific grammars, by reference to TCU position within a turn“ (ebd., S. 63, vgl. auch S. 76, 108-111). 
müssen; und gegenüber dem Begriff ,Kontextualisierungsverfahren‘ betont der Begriff ,Praktik` stärker und begrifflich offensichtlicher, dass diese Verwendung ein aktives Tun ist, das zudem in einem sequenziellen Kontext positioniert ist. Obwohl die Begriffe ,Praktiken' und ,Ressourcen' also eigentlich keine neuen Erkenntnisse reflektieren, sind sie im Kontext von CA und IL einfach nur die auch assoziativ geeigneteren.

Ohne die Analyse und Identifizierung von Praktiken könnten wir begrifflich nicht unterscheiden zwischen dem designierten, mit einer bestimmten Handlung assoziierten Gebrauch von Ressourcen, eben als routinisierte Praktik, und dem Gebrauch der gleichen Ressourcen in oder für andere als ihre designierten Handlungen, wo sie dann als nicht-erwartbare Ressourcen ggf. besondere Bedeutungen nahelegen oder Inferenzen auslösen. Für die präzise Beschreibung der Verwendung sprachlicher und anderer Ressourcen in ihren sequenziellen Kontexten brauchen wir also den Begriff ,Praktik‘.

\section{Literatur}

Christmann, Gabriela B./Günthner, Susanne (1996): Sprache und Affekt. Die Inszenierung von Entrüstung im Gespräch. In: Deutsche Sprache 24, S. 1-33.

Couper-Kuhlen, Elizabeth/Selting, Margret (2001): Introducing Interactional Linguistics. In: Selting, Margret/Couper-Kuhlen, Elizabeth (Hg.): Studies in Interactional Linguistics. Amsterdam, S. 1-22.

Couper-Kuhlen, Elizabeth/Selting, Margret (i.Vorb.): Interactional Linguistics.

Drew, Paul (1998): Complaints about transgressions and misconduct. In: Research on Language and Social Interaction 31, S. 295-325.

Drew, Paul/Holt, Elizabeth (1988): Complainable matters: The use of idiomatic expressions in making complaints. In: Social Problems 35, S. 398-417.

Goffman, Erving (1978): Response cries. In: Language 54, S. 787-815.

Goodwin, Charles (1984): Notes on story structure and the organization of participation. In: Atkinson, John M./Heritage, John (Hg.): Structures of social action. Cambridge, S. 225-246.

Goodwin, Marjorie Harness (1997): By-play: Negotiating evaluation in storytelling. In: Guy, Gregory R. et al. (Hg.): Towards a social science of language. Bd. 2: Social interaction and discourse structures. Amsterdam/Philadelphia, S. 77-102.

Goodwin, Marjorie Harness/Goodwin, Charles (2000): Emotion within situated activity. In: Duranti, Alessandro (Hg.): Linguistic anthropology: A reader. Malden, MA/Oxford, S. 239-257.

Gumperz, John (1982): Discourse strategies. Cambridge.

Günthner, Susanne (2000): Vorwurfsaktivitäten in der Alltagsinteraktion. Tübingen.

Günthner, Susanne (2006): Grammatische Analysen der kommunikativen Praxis - ,Dichte Konstruktionen' in der Interaktion. In: Deppermann, Arnulf/Fiehler, Reinhard/SpranzFogasy, Thomas (Hg.): Grammatik und Interaktion. Untersuchungen zum Zusammenhang von grammatischen Strukturen und Gesprächsprozessen. Radolfzell, S. 95-121. Internet: www.verlag-gespraechsforschung.de/2006/pdf/grammatik.pdf (Stand: 16.6.2015). 
Günthner, Susanne/Knoblauch, Hubert (1994): ,Forms are the food of faith‘ - Gattungen als Muster kommunikativen Handelns. In: Kölner Zeitschrift für Soziologie und Sozialpsychologie 46, S. 693-723.

Heritage, John (2010): Conversation Analysis: Practices and methods. In: Silverman, David (Hg.): Qualitative research: Theory, method and practice. 3. Aufl. London, S. 208-230.

Jefferson, Gail (1978): Sequential aspects of storytelling in conversation. In: Schenkein, Jim (Hg.): Studies in the organization of conversational interaction. New York, S. 219-248.

Jefferson, Gail (1979): A technique for inviting laughter and its subsequent acceptance declination. In: Psathas, George (Hg.): Everyday language. Studies in Ethnomethodology. New York u.a., S. 79-95.

Kotthoff, Helga (1995): Oral performance in interactional sociolinguistics: Funny stories among friends. In: Toegepaste Taalwetenschap in Artikelen 52, 2, S. 23-55.

Kotthoff, Helga (2006): Gender and humor. The state of the art. In: Journal of Pragmatics 1 , S. 4-26.

Kotthoff, Helga (2007): Oral genres of humor. On the dialectic of genre knowledge and creative authoring. In: Pragmatics 17, 2, S. 263-297.

Levinson, Stephen C. (2013): Action formation and ascription. In: Sidnell, Jack/Stivers, Tanya $(\mathrm{Hg}$.): The handbook of conversation analysis. Oxford, S. 103-130.

Local, John/Walker, Gareth (2008): Stance and affect in conversation: On the interplay of sequential and phonetic resources. In: Text \& Talk 28, S. 723-747.

Ochs, Elinor/Schieffelin, Bambi (1989): Language has a heart. In: Text \& Talk 9, S. 7-25.

Pomerantz, Anita (1986): Extreme case formulations: A way of legitimizing claims. In: Human Studies 9, S. 219-229.

Quasthoff, Uta (2001): Erzählen als interaktive Gesprächsstruktur. In: Brinker, Klaus et al. (Hg.): Text- und Gesprächslinguistik. 2. Halbbd.: Gesprächslinguistik. (= Handbücher zur Sprach- und Kommunikationswissenschaft 16.2). Berlin u.a., S. 1293-1309.

Reber, Elizabeth (2012): Affectivity in interaction. Sound objects in English. Amsterdam.

Sacks, Harvey (1971): Das Erzählen von Geschichten innerhalb von Unterhaltungen. In: Kjolseth, Rolf/Sack, Fritz (Hg.): Zur Soziologie der Sprache. Opladen, S. 307-314.

Sacks, Harvey (1986): Some considerations of a story told in ordinary conversation. In: Poetics 15, S. 127-138.

Sacks, Harvey/Schegloff, Emanuel A./Jefferson, Gail (1974): A simplest systematics for the organization of turn-taking for conversation. In: Language 50, S. 696-735.

Schegloff, Emanuel A. (1996): Confirming allusions: Toward an empirical account of action. In: American Journal of Sociology 104, S. 161-216.

Schegloff, Emanuel A. (1997): Practices and actions: Boundary cases of other-initiated repair. In: Discourse Processes 23, S. 499-545.

Schegloff, Emanuel A. (2000): On granularity. In: Annual Review of Sociology 26, S. 715-720.

Schegloff, Emanuel A. (2007): Sequence organization in interaction. A primer in conversation analysis. Bd. 1. Cambridge.

Selting, Margret (1995a): Prosodie im Gespräch. Aspekte einer interaktionalen Phonologie der Konversation. Tübingen.

Selting, Margret (1995b): Sprechstile als Kontextualisierungshinweise. Die sprechstilistische Kontextualisierung konversationeller Aktivitäten, am Beispiel mündlicher Erzählungen in Gesprächen. In: Gerhard Stickel (Hg.): Stilfragen. (= Jahrbuch des Instituts für deutsche Sprache 1994). Berlin/New York, S. 225-256. 
Selting, Margret (1996): Prosody as an activity-type distinctive signalling cue in conversation: The case of so-called, astonished questions" in repair-initiation. In: Couper-Kuhlen, Elizabeth/Selting, Margret (Hg.): Prosody in conversation. Interactional studies. Cambridge, S. 231-270.

Selting, Margret (2010): Affectivity in conversational storytelling: An analysis of displays of anger or indignation in complaint stories. In: Pragmatics 20, S. 229-277.

Selting, Margret (2012): Complaint stories and subsequent complaint stories with affect displays. In: Journal of Pragmatics 44, S. 387-415.

Selting, Margret (2013): Verbal, vocal, and visual practices in conversational interaction. In: Müller, Cornelia et al. ( $\mathrm{Hg}$.): Body - language - communication. An international handbook on multimodality in human interaction. Berlin u.a., S. 589-609.

Selting, Margret (i.Vorb.): The display and management of affect/emotion in social interaction. Illustrated with the construction of climaxes of joyful or amusing stories.

Selting, Margret et al. (2009): Gesprächsanalytisches Transkriptionssystem 2 (GAT 2). In: Gesprächsforschung - Online-Zeitschrift zur verbalen Interaktion 10, S. 353-402. Internet: www.gespraechsforschung-ozs.de/fileadmin/dateien/heft2009/px-gat2.pdf (Stand: 16.6.2015).

Selting, Margret/Couper-Kuhlen, Elizabeth (2001): Forschungsprogramm „Interaktionale Linguistik“. In: Linguistische Berichte 187, S. 257-287.

Sidnell, Jack (2006): Coordinating gesture, talk, and gaze in reenactments. In: Research on Language and Social Interaction 39, S. 377-409.

Sidnell, Jack (2010): Conversation Analysis. An introduction. Chichester.

Stivers, Tanya (2008): Stance, alignment, and affiliation during storytelling: When nodding is a token of affiliation. In: Research on Language and Social Interaction 41, S. 31-57.

Szczepek Reed, Beatrice (2014): Prosodic, lexical and sequential cues for assessments with German süß: Assemblages for action and public commitment. In: Barth-Weingarten, Dagmar/Szczepek Reed, Beatrice (Hg.): Prosodie und Phonetik in der Interaktion. Prosody and Phonetics in Interaction. Mannheim, S. 162-186. Internet: www.verlaggespraechsforschung.de/2014/pdf/szczepek-audio.pdf (Stand: 15.5.2015).

Wilkinson, Sue/Kitzinger, Celia (2006). Surprise as an interactional achievement: Reaction tokens in conversation. In: Social Psychology Quarterly 69, S. 150-182. 


\title{
Anhang
}

\section{Zusammenstellung der wichtigsten GAT2-Transkriptionskonventionen ${ }^{11}$}

\author{
Sequenzielle Struktur/Verlaufsstruktur \\ [ ] \\ Überlappungen und Simultansprechen \\ schneller, unmittelbarer Anschluss neuer Sprecherbeiträge oder \\ Segmente (latching)
}

Ein- und Ausatmen

$\begin{array}{ll}{ }^{\circ} \mathrm{h} / \mathrm{h}^{\circ} & \text { Ein-/Ausatmen von ca. 0,2-0,5 Sek. Dauer } \\ { }^{\circ} \mathrm{hh} / \mathrm{hh}{ }^{\circ} & \text { Ein-/Ausatmen von ca. 0,5-0,8 Sek. Dauer } \\ { }^{\circ} \mathrm{hhh} / \mathrm{hhh}^{\circ} & \text { Ein-/Ausatmen von ca. 0,8-1,0 Sek. Dauer }\end{array}$

Pausen

(.)

Mikropause, geschätzt, bis ca. 0,2 Sek.

$(-)$

kurze geschätzte Pause von ca. 0,2-0,5 Sek.

$(--)$

$(---)$ mittlere geschätzte Pause, ca. $0,5-0,8$ Sek.

(0.5) längere geschätzte Pause, ca. 0,8-1,0 Sek.

$(2.0)$ gemessene Pausen, ca. 0,5 bzw. 2,0 Sek. (Angabe mit einer Stelle hinter dem Punkt)

Segmentale Konventionen

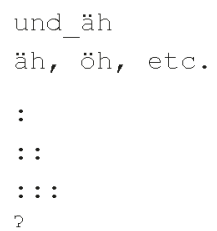

Lachen und Weinen

haha hehe hihi

((lacht)) ((weint))
Verschleifungen innerhalb von Einheiten Verzögerungssignale, sog. "gefüllte Pausen“

Dehnung, Längung, um ca. 0,2-0,5 Sek. Dehnung, Längung, um ca. 0,5-0,8 Sek. Dehnung, Längung, um ca. 0,8-1,0 Sek. Abbruch durch Glottalverschluss

einsilbige Signale

zweisilbige Signale

mit Glottalverschlüssen, meistens verneinend

silbisches Lachen

Beschreibung des Lachens

11 Siehe Selting et al. (2009) 
$\langle<$ lachend $>\quad>\quad$ Lachpartikeln in der Rede, mit Reichweite

$\langle<:-)\rangle$ soo $\quad$ "smile voice“"

Akzentuierung

$\begin{array}{ll}\text { akZENT } & \text { Fokusakzent } \\ \text { akzEnt } & \text { Nebenakzent } \\ \text { ak!ZENT! } & \text { extra starker Akzent } \\ \text { ak/ZENT } & \text { Beginn eines rhythmischen Fußes }\end{array}$

Tonhöhenbewegung am Ende von Intonationsphrasen

$\begin{array}{ll}? & \begin{array}{l}\text { hoch steigend } \\ \text { mittel steigend } \\ -\end{array} \\ \text { gleichbleibend } \\ \text { mittel fallend } \\ \text { tief fallend }\end{array}$

Auffällige Tonhöhensprünge

$\uparrow$

$\downarrow$

$\uparrow \uparrow$

$\downarrow \downarrow$

kleinere Tonhöhensprünge nach oben

kleinere Tonhöhensprünge nach unten

größere Tonhöhensprünge nach oben

größere Tonhöhensprünge nach unten

Verändertes Tonhöhenregister

$\begin{array}{lll}<<t> & > & \text { tiefes Tonhöhenregister } \\ <<h> & > & \text { hohes Tonhöhenregister }\end{array}$

Intralineare Notation von Akzenttonhöhenbewegungen

\begin{tabular}{|c|c|}
\hline so & fallend \\
\hline 'SO & steigend \\
\hline - SO & gleichbleibend \\
\hline & steigend-fallend \\
\hline so & fallend-steigend \\
\hline$\uparrow$ 、 & kleiner Tonhöhensprung hoch zum Gipfel der Akzentsilbe \\
\hline & kleiner Tonhöhensprung herunter zum Tal der Akzentsilbe \\
\hline$\uparrow^{{ }^{-} \text {SO bzw. } \downarrow^{-} \text {SO }}$ & $\begin{array}{l}\text { Tonhöhensprünge zu auffallend höheren bzw. tieferen gleichblei- } \\
\text { benden Akzenten }\end{array}$ \\
\hline$\uparrow \uparrow$ 'so bzw. $\downarrow \downarrow$ 'so & $\begin{array}{l}\text { auffallend hohe bzw. tiefe Tonhöhensprünge zum Gipfel bzw. Tal } \\
\text { der Akzentsilbe }\end{array}$ \\
\hline
\end{tabular}

Lautstärke- und Sprechgeschwindigkeitsveränderungen, mit Extension

\begin{tabular}{|c|c|c|}
\hline$\langle\langle\mathrm{E}\rangle$ & $>$ & $=$ forte, laut \\
\hline$<<f f>$ & $>$ & $=$ fortissimo, sehr laut \\
\hline$\langle\langle p\rangle$ & $>$ & = piano, leise \\
\hline cpp> & $>$ & $=$ pianissimo, sehr leise \\
\hline alls & $>$ & = allegro, schnell \\
\hline$\langle 1 \in n>$ & $>$ & $=$ lento, langsam \\
\hline rescl & $>$ & $=$ crescendo, lauter werdend \\
\hline im> & $>$ & $=$ diminuendo, leiser werdend \\
\hline
\end{tabular}




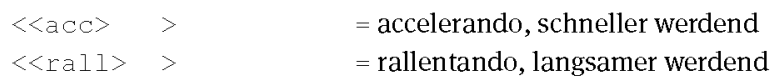

Veränderung der Stimmqualität und Artikulationsweise

<<creaky> > glottalisiert, „Knarrstimme“

$<<f l u ̈ s t e r n d>\quad>\quad$ Beispiel für Veränderung der Stimmqualität

Sonstige Konventionen

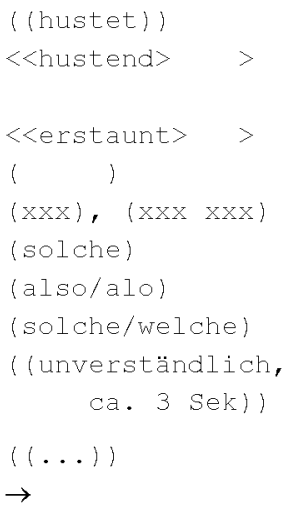

para-/außersprachliche Handlungen $u$. Ereignisse

sprachbegleitende para- und außersprachliche Handlungen und

Ereignisse mit Reichweite

interpretierende Kommentare mit Reichweite

unverständliche Passage ohne weitere Angaben

ein bzw. zwei unverständliche Silben

vermuteter Wortlaut

mögliche Alternativen

unverständliche Passage mit Angabe der Dauer

Auslassung im Transkript

Verweis auf im Text behandelte Transkriptzeile 